\title{
The formation of martensite crystals with a degenerate structure of transformation twins
}

\author{
M. P. Kashchenko ${ }^{1,2, \dagger}$, N. M. Kashchenko ${ }^{1}$, V. G. Chashchina ${ }^{1,2}$ \\ †mpk46@mail.ru \\ ${ }^{1}$ B.N. Yeltsin Ural Federal University named after the first President of Russia, 19 Mir str., Yekaterinburg, 620002, Russia \\ ${ }^{2}$ Ural State Forest Engineering University, 37 Sybirskyi Tract str., Yekaterinburg, 620100, Russia
}

\begin{abstract}
The dynamic theory of martensitic transformation explains the phenomenon of initiation of the fine structure of transformation twins as a result of the coordinated action of relatively long-wave displacements ( $\ell$-waves) with more short-wave displacements ( $s$-waves). Being a part of a controlling wave process, $\ell$-waves provide habit formation, whereas $s$-waves play the leading part in initiation of the main component of twin structure (TS). It was shown that the dynamic theory allows one to consider the degenerate TS (DTS) formation as a particular case of TS when the twin component volume is converted to zero. In this work the case of DTS is discussed by the example of crystals with habits $\{110\}$. The peculiarity of this variant consists in the fact that in order to describe the morphology of transformation it is enough to only consider longitudinal waves running along axes $<100>$ as a part of the controlling wave process. In particular, habit (101) may be matched with a pair of $\ell$-waves with velocities along [100] and [001] and a pair of $s$-waves with velocities along [100] and [010]. At the same time, condition $d_{s}=\lambda_{s} / 4$, where $\lambda_{s}$ is the wavelength of $s$-waves, and $d_{s}$ is a transversal (in directions [100] and [010]) size of the initial exited (oscillatory) $s$ - $\ell$ - cell with longitudinal size $d_{s}<d_{\ell}<\lambda_{\ell} / 2$, conforms to DTS formation. For martensite transformations fcc-bct, bcc-fct, fcc$\mathrm{fct}$, the transition to finishing deformations and the connection of the values of tetragonality of martensite and volume effect with one of characteristic main values of deformation tensor are discussed.
\end{abstract}

Keywords: martensitic transformation, controlling process, twin structure, tetragonality, volume effect.

\section{Introduction}

The formation of martensite crystals possessing a fine inner twin structure (TS) is rather typical of martensite transformations (MT): from pronounced transitions of the first type (i.e., in disordered iron-based alloys [1-4]) to thermo-elastic transitions (i.e., in alloys based on titanium nickelide and many non-ferrous alloys [5-8]). Transformation twins, as a rule, represent a combination of interchanging areas with an orthogonal (at the initial phase) orientation of main axes of deformations. The crystal geometry analysis [9-11] attributes twinning to non-homogeneous deformation necessary to maintain the macroscopic invariance of the habit plane. The prescribed habit orientation is provided by the strictly defined ratio $\beta$ of the main and twin components of TS.

However, experiments [12] have shown that $\beta$ magnitude can vary within the limits of one crystal, and in [13-14] non-twinned thin-plated crystals or streaks of lens-shaped crystals were observed.

In the dynamic theory of MT [15 - 18], the habit formation is determined by the action of relatively long-wave shifts ( $\ell$-waves), and the formation of TS takes place considering relatively short-wave shifts ( $s$-waves) in the controlling wave process (CWP). Thus, a habit orientation does not depend on $\beta$ magnitude. The model of formation of a regular TS [17 - 18] with coordinated action of $s$ - and $\ell$-waves is taken as a basic one. Origination of waves (in the form of a wave bundle) is provided by initial excited (oscillatory) states (IES). It should be taken into account that $\ell$-waves originate in the form of elongated rectangular parallelepipeds in the areas designated by the elastic fields of dislocation centers of initiation, and $s$-waves are generated by spontaneously initiating excited cells at the optimal (for MT realization) ratio of $\ell$ - and $s$ - oscillation phases.

The main component of TS is physically isolated, namely it is initiated mainly by $s$-waves action, whereas the twin component appears as an interlayer between the nearest main components (due to the coherent link of contacting areas of the lattice), i.e. the process of its formation has a subordinate character. Wave vectors of longitudinal $s$-waves are directed along the orthogonal axis of symmetry of the 4-th order $<100>$ of the initial phase. In the area of superposition of $s$-waves front, the plane deformation of the expansioncontraction type is initiated. In a pair of quasi-longitudinal $\ell$-waves, the first wave provides Bain expansion along the third axis from $<100>$ family, common for both components of TS. The second $\ell$-wave initiates contraction deformation, simultaneously marking out one of the axes $<100>$ as main contraction axis of basic component of TS, which forms the minimal angle with direction $\boldsymbol{n}_{2 \ell}$ of its wave vector. The induced reproduction of the excited $s$-cell (initially originated spontaneously) in the course of CWP propagation suggests that $s$-cell nearest to the initial one appears after passing the two legs of a triangle in directions [1 10$]$ and [110] by superposition of $s$-waves within the same time period, 
and the hypotenuse of the triangle at the speed equal to $v_{2 \ell}{ }^{\prime}$-projection to the plane (001) at the velocity of $\ell$-wave $v_{2 \rho}$, bearing compression deformation (Fig. 1).

Suppose that two legs of this triangle are passed at $Z$ speed $\sqrt{2} v_{s \Delta}$, we get the condition:

$$
v_{s \Delta}=v_{2 \ell}{ }^{\prime} \cos \psi
$$

where $\psi$ is an acute angle between $\boldsymbol{v}_{2 \ell}{ }^{\prime}$ and $\left.\boldsymbol{v}_{s \Delta} \|<001\right\rangle_{\gamma}$. In terms of a harmonic description of a threshold deformation, it is considered (as is seen from Fig. 1) that the loss of stability of the lattice of the initial phase corresponding to the main component of TS takes place in the area of transversal size

$$
d_{s}<\lambda_{s} / 2, \tilde{d}_{s}=d_{s} / \lambda_{s}<1 / 2 .
$$

Then it is evident that, for the proportion of TS components, the equality holds:

$$
\beta_{t w}=\tilde{d}_{s} /\left(1+\operatorname{tg} \psi-4 \tilde{d}_{s}\right) .
$$

The model of regular TS formation serves as a base for passing to the description of real distributions of transformation twins, as a rule, possessing fragmentation, and each fragment being related to its own spontaneously initiated active cell [19-21].

However, this work studies an extreme case of the degenerate twin structure (DTS) following on from the resultsof the dynamic theory for regular TS. It should be noted that from the standpoint of minimizing the elastic energy, the transition from a twin to a monodomain structure was briefly discussed in [22].

\section{The dynamic theory of formation of martensite crystals with degenerate twin structure}

According to (3), the case of DTS corresponds to the condition:

$$
1+\operatorname{tg} \psi-4 \tilde{d}_{s}=0 .
$$

Next we suppose that the wave process transfers the threshold deformation initiating fcc-bcc (bct) martensite transformation with Bain type of deformation. Then a simple variant of CWP model includes a pair of $\ell$-waves; one of them propagates along [001], bears expansion deformation, and is purely longitudinal, while the other quasi-longitudinal wave bears compression deformation and has a wave vector in plane (110) making angle $\psi$ with [100]. A pair of $s$-waves is similar to those shown in Fig. 1, they propagate along orthogonal directions of $\Delta$-axes of symmetry of the fourth order and bear deformation of the «expansion-compression» type coordinated with $\ell$-waves. From (1) we can find for two extreme $\psi$ values:

$$
\Psi=0 \rightarrow \tilde{d}_{s}=1 / 4, \quad \Psi=\pi / 4 \rightarrow \tilde{d}_{s}=1 / 2 .
$$

The comparison with (5) shows that realization of the case of the second wave propagation strictly along the direction of $\Sigma$-axis of symmetry of the second order $(\Psi=\pi / 4)$ would mean conversion of the contribution to threshold compression deformation from $\ell$-wave at boundary habit planes to zero. However, even for small deviations of $\Psi$ from $\pi / 4$ the inequality (2) is met. Besides, coordination of the velocities of $s$ - и $\ell$-waves propagation (1) requires the equality

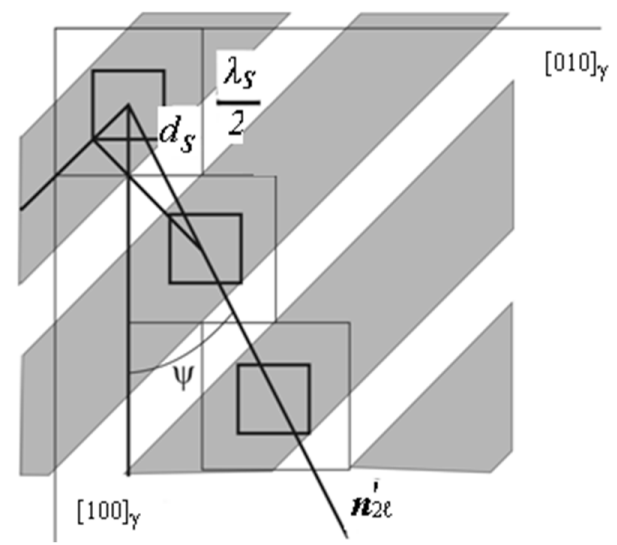

Fig. 1. The dynamic model of formation of a regular layer (in particular, a twin one) structure with the 2:1 proportion of TS components [18].

(6) to be met with $\Psi=\pi / 4$ :

$$
v_{\ell \Sigma} / v_{s \Delta}=æ_{\ell s}=\sqrt{2} .
$$

Note that if $v_{\ell \Sigma}, v_{s \Delta}$ are interpreted as group velocities of $s$ - and $\ell$-waves from different diapasons of wave vectors, the condition (6) can always be met since $v_{s \Delta}$ monotonously decreases to zero at the boundary of Brilluen zone (from maximum values in the area where dispersion can be ignored).

On the contrary, in the extreme case of $\Psi=0$ the inequality (4) is met. Correlation (1) takes the form:

$$
v_{\ell \Delta} / v_{s \Delta}=æ_{\ell s}=1 \text {. }
$$

Obviously, condition (7) is easily met in the area of wave vectors, where it is possible (with satisfactory accuracy) to ignore the difference of velocities due to the dispersion (to wave vectors $q \sim 0.1 q_{\max }=0.2 \pi / a$, where $a$ is the parameter of the lattice of the initial phase).

Recall that normal $N$ to habit plane in the dynamic theory of martensite transformation comes to linear combination of the pair of $\ell$-waves velocities:

$$
\begin{gathered}
N_{1,2 \ell} \| n_{2 \ell} \pm æ_{\ell \ell} n_{1 \ell}, æ_{\ell \ell}=\frac{v_{2 \ell}}{v_{1 \ell}}, \\
\left|n_{1,2 \ell}\right|=1, \quad n_{1 \ell}=\frac{v_{1 \ell}}{v_{1 \ell}}, \quad n_{2 \ell}=\frac{v_{2 \ell}}{v_{2 \ell}} .
\end{gathered}
$$

Then, in case of $\Psi=0$ we have:

$$
\mathfrak{x}_{\ell \ell}=1, \quad \boldsymbol{n}_{1 \ell}=[001], \quad \boldsymbol{n}_{2 \ell}=[100], \quad \boldsymbol{N}_{1,2 \ell} \|[101],[10 \overline{1}] .
$$

If $\Psi=\pi / 4$ and $æ_{\ell \ell}=\sqrt{2}$, then

$$
\boldsymbol{n}_{1 \ell}=[001], \quad \boldsymbol{n}_{2 \ell}=\frac{1}{\sqrt{2}}[110], \quad \boldsymbol{N}_{1,2 \ell} \|[112],[\overline{1} \overline{1} 2] .
$$

Note that «hidden» orientations of normals

$$
N_{1,2 s} \|[110],[1 \overline{1} 0]
$$

to planes of twinning can be trivially found from (8) if we change:

$$
æ_{\ell \ell} \rightarrow æ_{s s}=1, \quad \boldsymbol{n}_{1 \ell} \rightarrow \boldsymbol{n}_{1 s}=[010], \quad \boldsymbol{n}_{2 \ell} \rightarrow \boldsymbol{n}_{2 s}=[100] .
$$


Strictly speaking, the removal of degeneracy by orientations of twinning planes suggests realization of transfer by values $\psi$ : either $\psi \leq \pi / 4$ or $\psi \geq \pi / 4$.

Then we confine ourselves to the extreme case of crystals with DTS and habits (110) allowing us to establish a simple relation between tetragonality of $\mathrm{t}$-martensite and one of the main values of the tensor of Bain deformation.

\subsection{Tetragonality of martensite with habits (110), at fcc-bct transformation}

We suppose that fcc-bct $(\gamma-\alpha)$ martensite transformation (MT) is realized and crystals with DTS and habits (110), originate. Recall that in the dynamic theory of MT, the cinematic description of a habit in the threshold mode complies with the deformation description. The requirement of coincidence of these descriptions means that the proportion of compression modulus $\varepsilon_{2}<0$ and tension modulus $\varepsilon_{1}>0$ in the threshold mode coincides with the ratio of squares of wave velocities:

$$
\frac{\varepsilon_{1}}{\left|\varepsilon_{2}\right|}=æ_{\ell \ell}^{2}
$$

Then it is not unreasonable to assume that austenite lattice having lost its stability and relaxing to new stable positions of atoms keeps the ratio of deformations in the diapason from the threshold to the final values. We also suppose that the first quick stage of transformation is linked to short-wave $s$-deformations (within time interval $T_{s} / 2$ of the order of the period of $s$-oscillations), and at this stage the finish (Bain $\varepsilon_{1 \mathrm{~B}}$ ) expansion deformation in $[010]_{y}$ direction $\varepsilon_{1 s}=\varepsilon_{1 \mathrm{~B}}>0$ is achieved. Since, the compression deformation along [100] at the first stage amounts to value $\varepsilon_{2 s}=-\varepsilon_{1 s}=-\varepsilon_{1 B}$. Since at the second stage (within time interval $T_{\ell} / 2$ of the order of the period of $\ell$-oscillations) compression and expansion are also carried out by waves with the same velocities, the deformation contribution is $\varepsilon_{2 \ell}=-\varepsilon_{1 \ell}$. It follows from the symmetry of bct lattice that expansion deformation $\varepsilon_{1 \ell}$ along [001] axis is equal to deformation $\varepsilon_{1 s}=\varepsilon_{1 \mathrm{~B}}$. Consequently, $\varepsilon_{2 \ell}=-\varepsilon_{1 \mathrm{~B}}$, i.e. at the second stage the contribution of $\ell$-wave in the main Bain compression deformation is equal to the contribution of $s$-wave at the first stage. Let's take into consideration that due to inequality $T_{\ell} \gg T_{s}, \ell$-compression affects the already collapsed lattice. As a result, linear dimension $a$ (a parameter of lattice fcc of austenite) in direction [100] decreases by $\left(1-\varepsilon_{1 \mathrm{~B}}\right)^{2}$ times, while lateral dimension $a / \sqrt{2}$ increases by $\left(1+\varepsilon_{1 \mathrm{~B}}\right)$ times. It is evident that to determine $t$ the ratio between the edge of the cube of the initial elementary cell of fcc lattice after compression deformation and the half of the diagonal of the cube face after expansion deformation should be used.

Therefore, the value of tetragonality is preset by expression:

$$
t_{\gamma-\alpha}=\left(1-\varepsilon_{1 \mathrm{~B}}\right)^{2} /\left[\left(1+\varepsilon_{1 \mathrm{~B}}\right) \sqrt{2}\right]
$$

According to $(14)$, the value $\varepsilon_{1 \mathrm{~B}}^{*} \approx 0.11290293 \approx 0.1129$ meets the condition of the absence of tetragonality $\left(t_{\gamma-\alpha}=1\right)$, $t_{\gamma-\alpha}>1$ at $\varepsilon_{1 \mathrm{~B}}<\varepsilon_{1 \mathrm{~B}}^{*}$ and $t_{\gamma-\alpha}<1$ at $\varepsilon_{1 \mathrm{~B}}>\varepsilon_{1 \mathrm{~B}}^{*}$. Note that the value of lattice tetragonality when analyzing martensite transformation is a convenient parameter normally reflecting the existence of certain regulations both in implantation alloys (i.e., carbon spreading about octahedral interstices in steels [23]), and in substitution alloys (i.e., normalization of atoms in alloys of stoichiometric composition, magnetic normalization [24]).

\subsection{The tetragonality of martensite with habits (110) at bcc-fct transformation}

We suppose that bcc-fct $(\alpha-\gamma)$ martensite transformation is realized and crystals with DTS and habits $(110)_{\alpha}$ originate. The main axis of extension is oriented along the direction $[010]_{\alpha} \|[001]_{\gamma}$ (the edge of bcc cell), and the main axes of compression are directed along the diagonals of bcc cell faces.

We suppose that the ultimate (Bain) compression deformation $\varepsilon_{2 B}$ is achieved at once, at the first shortwave stage. Then, making an examination similar to the onementioned above, after $\ell$-compression we have:

$$
t_{\alpha-\gamma}=\left(1+\left|\varepsilon_{2 \mathrm{~B}}\right|\right)^{2} /\left[\left(1-\left|\varepsilon_{2 \mathrm{~B}}\right|\right) \sqrt{2}\right]
$$

instead of formula (14).

According to (15), the absence of tetragonality $\left(t_{\alpha-\gamma}=1\right)$ is fulfilled by the value $\left|\varepsilon_{2 B}^{*}\right| \approx 0.117291 \approx 0.1173, t_{\alpha-\gamma}<1$ at $\left|\varepsilon_{2 \mathrm{~B}}\right|<\left|\varepsilon_{2 \mathrm{~B}}^{*}\right|$ and $t_{\alpha-\gamma}>1$ at $\left|\varepsilon_{2 \mathrm{~B}}\right|>\left|\varepsilon_{2 \mathrm{~B}}^{*}\right|$. For instance, according to [24], tetragonality $t_{\alpha-\gamma} \approx 0.98$ is observed in crystals of martensite of alloy $\mathrm{Cu}-38.55$ mas. $\% \mathrm{Zn}$ with habits $\left\{\begin{array}{lll}2 & 11 & 12\end{array}\right\}_{\alpha}$ close to $\{110\}_{\alpha}$, which is possible (in concordance with (15)) at $\varepsilon_{2 \mathrm{~B}} \approx 0.11$.

\subsection{The tetragonality of martensite with habits (110) $)_{\alpha}$ under fcc-fct transformations}

Transformations fcc-fct are observed in alloys under thermoelastic martensite transformations. Depending on the material, deformation values can vary within a rather wide range. Therefore, in alloys $\mathrm{Fe}$-Pt with compositions close to stoichiometric $\mathrm{Fe}_{3} \mathrm{Pt}$, transformation features depend on the degree of normalization and are characterized by the decrease of deformation values, temperature hysteresis, specific volume change during normalization progress. In alloys $I n-T l$, a transformation exists as a transition of the I-st kind close to a transition of the II-nd kind [1, 25], as a rule, the crystals are twinned, and their habits belong to the family $\{110\}_{\alpha}$.

The edges of a fcc cell are naturally chosen as the main axes of deformation. If the extension deformation dominates, then $t>1$, the predominance of compression leads to $t<1$, $t=1$ only in the absence of deformation:

$$
\begin{gathered}
t=\left(1+\varepsilon_{1}\right)^{2} /\left(1-\varepsilon_{1}\right) \rightarrow t>1, \\
t=\left(1-\left|\varepsilon_{2}\right|\right)^{2} /\left(1+\left|\varepsilon_{2}\right|\right) \rightarrow t<1 .
\end{gathered}
$$

In record (16), as well as in (14) and (15), habit (110) and degenerate twin structure are considered.

\section{Discussion}

In the above stated diagrams of formation of crystals with habit (101) and DTS of the corresponding wave bearer of three-dimensional Bain deformation were used. However, 
options with a wave bearer of two-axes (plane) deformation leading to a fastest transformation of the plane in which the main deformation axes lie should also be discussed. At the same time it is supposed that in the direction orthogonal to isolated plane, homogeneous deformation recovering the symmetry of lattice takes place due to the electron correlations.

\subsection{The tetragonality of martensite in the case of wave bearer of plane deformation}

In the case of fcc-fct, martensite transformation with habit (101), planes (010), are deformed and homogeneous extension along $[010]_{\gamma}$ is supposed. Such mechanism does not require two stages of transformation. Therefore, under equal-in-size deformations of extension and compression for tetragonality instead (14) we have correlation:

$$
\begin{gathered}
\tilde{t}_{\gamma-\alpha}=\sqrt{2}\left(1-\varepsilon_{1 \mathrm{~B}}\right) /\left(1+\varepsilon_{1 \mathrm{~B}}\right), \\
\varepsilon_{1 \mathrm{~B}}^{*}=(\sqrt{2}-1) /(\sqrt{2}+1) \approx 0.1716, \\
\varepsilon_{1 \mathrm{~B}}<\varepsilon_{1 \mathrm{~B}}^{*} \rightarrow \tilde{t}_{\gamma-\alpha}>1, \\
\varepsilon_{1 \mathrm{~B}}>\varepsilon_{1 \mathrm{~B}}^{*} \rightarrow \tilde{t}_{\gamma-\alpha}<1, \\
\varepsilon_{1 \mathrm{~B}}=\varepsilon_{1 \mathrm{~B}}^{*} \rightarrow \tilde{t}_{\gamma-\alpha}=1 .
\end{gathered}
$$

Similarly, for the case of bcc-fct transformation instead of (15) we find expression for $t$ reciprocal to ratio (17):

$$
\begin{gathered}
\tilde{t}_{\alpha-\gamma}=\left(1+\left|\varepsilon_{2 \mathrm{~B}}\right|\right) /\left[\left(1-\left|\varepsilon_{2 \mathrm{~B}}\right|\right) \sqrt{2}\right], \\
\left|\varepsilon_{2 \mathrm{~B}}^{*}\right|=(\sqrt{2}-1) /(\sqrt{2}+1) \approx 0.1716, \\
\left|\varepsilon_{2 \mathrm{~B}}\right|<\left|\varepsilon_{2 \mathrm{~B}}^{*}\right| \rightarrow \tilde{t}_{\alpha-\gamma}>1, \\
\left|\varepsilon_{2 \mathrm{~B}}\right|>\left|\varepsilon_{2 \mathrm{~B}}^{*}\right| \rightarrow \tilde{t}_{\alpha-\gamma}<1, \\
\left|\varepsilon_{2 \mathrm{~B}}\right|=\left|\varepsilon_{2 \mathrm{~B}}^{*}\right| \rightarrow \tilde{t}_{\alpha-\gamma}=1 .
\end{gathered}
$$

Obviously, the variant of plane deformation under fccfct transformation corresponds to the removal of squares relationship in numerators of formulas (16):

$$
\begin{gathered}
\tilde{t}=\left(1+\varepsilon_{1}\right) /\left(1-\varepsilon_{1}\right) \rightarrow \tilde{t}>1, \\
\tilde{t}=\left(1-\left|\varepsilon_{2}\right|\right) /\left(1+\left|\varepsilon_{2}\right|\right) \rightarrow \tilde{t}<1 .
\end{gathered}
$$

According to (21), $\tilde{t}>1$ if expansion deformation dominates; $\tilde{t}<1$ if compression dominates, and $\tilde{t}=1$ only if there is no deformation.

It should also be noted that high symmetry of habits of $\{110\}$ family, as well as the attribution of typical orientations of the bounds of transformation twins (in the case of crystals with DTS) to this family may result in pairs of crystals with the same habits, but with orthogonal orientations of dominant deformations. It means that crystals will have twin orientations with incongruous inner planes of twinning (though hidden).

Evedently, according to (5) the realization of requirement $\tilde{d}_{s}=1 / 4$ as a regular condition (for crystals with DTS and habits (110)) is very rigid. Nevertheless, such variants can be realized.

Making precise comparison of the calculated and experimentally observed values of martensite tetragonality one needs to also consider that main axes of $s$-deformation undergo rotation losing their initial orthogonality. Therefore, $s$-and $\ell$-deformations have to be calculated using the formulas for plane deformation. This issue is discussed in detail in [15], and we use the simplified variant of formulas for tetragonality to reveal their principal simplicity and obviousness. Indeed, the relation between ratios of deformations and wave velocities (13) permits the association of " $t$ " magnitude with one of the main values of Bain deformation that can be easily used as a phenomenological parameter when processing experimental data. Its magnitude can be found using the measurement data of the parameters of lattices of the initial and ultimate phases. The value of that parameter can be found from the condition of the minimum thermodynamic potential (corresponding to the experimental conditions), the main part played by the contribution of zone energy of electrons (in the case of martensite transformation in metals and alloys).

At the same time, there is a sufficient difference of " $t$ " magnitudes in cases of wave bearers of three-dimensional and plane deformations. Reliable separation of the pointed variants by " $t$ " magnitude even disregarding the measurement of orientations of axes after the first stage of $s$-deformation is also allowed.

\subsection{A relative change of volume $\delta$}

Alhough more attention was paid to the orientations of habits and martensite tetragonality, the dynamic theory permit the establishment of a representative list of morphological features including orientation ratios, macroshift, relative change of volume $\delta$.

In particular, at $\gamma-\alpha$ MT in the same approximation as for (14), we easily find:

$$
\delta_{\gamma-\alpha}=\left(1-\varepsilon_{1 \mathrm{~B}}^{2}\right)^{2}-1=-\varepsilon_{1 \mathrm{~B}}^{2}\left(2-\varepsilon_{1 \mathrm{~B}}^{2}\right) \approx-2 \varepsilon_{1 \mathrm{~B}}^{2}<0 .
$$

This means that for real values $\varepsilon_{1 \mathrm{~B}} \sim 0.1$ the volume effect is anomalous in sign (usually for $\gamma-\alpha$ MT $\delta>0$ ), but with a typical value (near 0.02). It is evident that origination of such crystals as a kind of relaxation «layers» between crystals with positive values of $\delta$ is quite admissible.

A similar result is obtained at $\alpha-\gamma$ MT, but in formula (22) change $\varepsilon_{1 \mathrm{~B}} \rightarrow\left|\varepsilon_{2 \mathrm{~B}}\right|$ has to be made.

$$
\delta_{\alpha-\gamma}=\left(1-\varepsilon_{2 \mathrm{~B}}^{2}\right)^{2}-1=-\varepsilon_{2 \mathrm{~B}}^{2}\left(2-\varepsilon_{2 \mathrm{~B}}^{2}\right) \approx-2 \varepsilon_{2 \mathrm{~B}}^{2}<0 .
$$

It is obvious that for fcc-fct transformation $\delta$ is calculated using formula (22) if $t>1$, and formula (23) if $t<1$.

Since in the extreme case of DTS under consideration the volume effects $\delta_{\gamma-\alpha}, \delta_{\alpha-\gamma}$ and $\delta$ are negative, the preferable option for its realization is connected with the direct MT occurring under cooling. Note that for the studied option of habits $\{110\}$ (in the bases of initial phases) the negative volume effect is a necessary (but insufficient) indicator of the mechanism of transformation with DTS.

According to the results of preliminary estimations, MT B2 $\rightarrow L 1_{0}$ observed under cooling of alloy $N i_{50} M n_{50}$ within the temperature interval of $M_{s}=980 \mathrm{~K}$ and $M_{f}=920 \mathrm{~K} \mathrm{[26]} \mathrm{can} \mathrm{be}$ attributed to such an extreme case. Without a detailed analysis it can be noted that for such MT the features expected for crystals with DTS are attained. Those are: packets of crystals twinned in couples (with the same habits $\{110\}_{\alpha}$ ); $\delta_{\alpha-\gamma}<0$, $\left|\delta_{\alpha-\gamma}\right| \sim 0.01$; magnitude of $t \approx 0.9$ in $M_{s}$ surroundings are typical of and in accord with value $\delta_{\alpha-\gamma}$. The observed system of fine secondary nanotwins does not preclude the description of such martensite as crystals with a degenerate "primary» 
structure of transformation twins.

Let's note that the variant with a wave bearer of plane deformation satisfies the abnormally high (at $\varepsilon_{1 \mathrm{~B}} \sim\left|\varepsilon_{2 \mathrm{~B}}\right| \sim 0.1$ ) values of relative volume changes:

$$
\begin{gathered}
\tilde{\delta}_{\gamma-\alpha}=\varepsilon_{1 \mathrm{~B}}\left(1-\varepsilon_{1 \mathrm{~B}}-\varepsilon_{1 \mathrm{~B}}^{2}\right) \approx \varepsilon_{1 \mathrm{~B}}>0, \\
\tilde{\delta}_{\alpha-\gamma}=-\left|\varepsilon_{2 \mathrm{~B}}\right|\left(1+\left|\varepsilon_{2 \mathrm{~B}}\right|-\left|\varepsilon_{2 \mathrm{~B}}\right|^{2}\right) \approx-\left|\varepsilon_{2 \mathrm{~B}}\right|<0 .
\end{gathered}
$$

In (25) and (26) nomination $\tilde{\delta}$, as formerly $\tilde{t}$, was introduced to distinguish the volume effect in the case of a wave bearer of plane deformation.

Using correlations (19) and (26) it evident that the abovementioned variant of transformation in $N i_{50} M n_{50}$ [26] is not connected with the bearer of plane deformation. Indeed, inserting value $\tilde{t}_{\alpha-\gamma}=0.9$ in (19), we obtain deformation $\left|\varepsilon_{2 \mathrm{~B}}\right| \approx 0.12$, at which, according to $(26), \tilde{\delta}_{\alpha-\gamma} \approx-0.12$ sufficiently exceeds the value observed.

Unless comparatively high values of $\tilde{\delta}$, the origination of such crystals in the composition of accommodated crystal ensembles does not contradict any condition. Especially in the case of transformations in austenite grains with diameters of submicroscopic and nano-diapasons when small sizes of crystals permit to avoid large deviations from the coherent conjugation of lattices of various phases.

\subsection{Additional remarks}

The discussed variant of transformation with DTS as additional to the commonly accepted variant of shift reconstruction associated with the softest transverse mode. A combined scenario when at the first stage longitudinal waves quickly form the fine inner central part of the crystal initiating the shift, which develops and causes a preferably thermo-elastic widening of crystals in the process of cooling, is quite possible.

Nano-twins inside crystals twinned in couples are conditionally attributed to «secondary» ones, i.e. not realized as «primary» transformation twins. Nevertheless, their appearance as transformation twins (inside the "primary» main components of DTS closing down) is possible due to the disturbance of the ratio for velocities (1). Really, due to the dispersion of phonon velocities the inequality $v_{s \Delta}<v_{\ell \Delta}$ is held, causing the initiation of irregular TS [19]. In connection with the possibility mentioned it would be more correct to use the submission of degenerate primary twin structure (DPTS) for such crystals.

In the general case, within the limits of one sample, different dynamic variants of transformation can be realized. For instance, symbiosis of crystals with habits $\{557\}$ and $\{225\}$ in steels is well known [23]. There are visible morphological variations for crystals with habits $\{334\}$ and $\{443\}$ in titanium as well [1]. Beside the above mentioned mechanisms, there is a variant of the fastest transformation of planes with their further reshuffle $[27,28]$. Such variability may be caused by local variations of chemical composition, appearance of additional variants of dislocation nucleation center (DNC) in the process of martensite crystals formation, short-wave fluctuations in CWP structure. The analysis of the variety of the observed morphological features to a substantial degree permits the reconstruction of the dynamic mechanism of MT. At the same time, the more complete the analyzed variety is, the more detailed the reconstruction of the dynamics of the process appears to be. One of the most remarkable examples is the modulation of TS including the data on the extreme variant of DTS.

As to the perspectives of the further research, it is advisable, first of all, to analyze the mechanism of formation of crystals with habits close to $\{h h \ell\}_{\gamma}$ as variants of transformation with DTS. In particular, the above mentioned case of planes $\{11 \sqrt{2}\}_{\gamma}$ correlating to habits $\{557\}_{\gamma}$ of the packet martensite at $\gamma-\alpha$ MT in ferrous alloys is of interest. Note that relations (3) and (4) also permit thestudy of crystals with habits $\{\mathrm{hk \ell}\}$ as variants with DTS. There, material parameters $æ_{\ell \ell}$ и $æ_{\ell s}$ will obviously play a sufficient part.

\section{Conclusion}

The variant of realization of martensite transformation with the degenerate structure of transformation twins presented in the work is an extreme case of the dynamic formation of twinned martensite crystals. At the same time, there is a possibility of wave description of three-dimension deformation of Bain type.

The description of crystals with DTS with habits $\{110\}$ looks most simply. The analysis carried out permits to attribute to this type, crystals of $L 1_{0}$-martensite originating when well-ordered NiMn alloy of equi-atomic composition is cooled.

Generalization and distribution of the methodology studied concerning the case of crystals with habits of general type advances the facilities for reconstruction of peculiarities of the controlling wave process basing of the information about the collection of morphological features observed.

\section{References}

1. B. A. BiIby, J. W. Christian, Martensitic Transformations. The Mechanism of Phase Transformations in Metals. The Institute of Metals. London (1956).

2. S. Miyazaki, K. Otsuka, C. M. Wayman. Acta Metall. 377, 1873 (1989).

3. M.P. Kashchenko, V.G. Chashchina, Phys. Usp. 54(4), 331 (2011). DOI: 10.3367/UFNr.0181.201104a.0345

4. M.P. Kashchenko, V. G. Chashchina. Letters on materials. 4(4), 307 (2014). DOI: 10.22226/2410-3535-2014-4-307-314

5. T. Maki, C. M. Wayman. Proc. 1st JIM Int. Symp. on New Aspects of Martensitic Transformation, Suppl. Trans. JIM. 17, 69 (1976).

6. V.M. Schastlivtsev, Yu. V. Kaletina, E.A. Fokina. Martensitic Transformations in Magnetic Field. Ekaterinburg, UrO RAN (2007) 323 p. (in Russian)

7. M.S. Wechsler, D. S. Lieberman, T. A. Read. Trans. AIME. 197, 1503 (1953).

8. J.S. Bowles, J. K. Mackenzie. Acta Metall. 2, 129 (1954).

9. J. S. Bowles, J. K. Mackenzie. Acta Metall. 2, 138 (1954).

10. J.S. Bowles, J. K. Mackenzie. Acta Metall. 2, 224 (1954).

11. A. B. Greninger, A. R. Troiano. Metal. Trans. 185, 590 (1949).

12. K. M. Knowles, D. A. Smith. Acta metall. 29, 101 (1981). DOI: 10.1016/0001-6160(81)90091-2 
13. M.P. Kashchenko, V.G. Chashchina, S.V. Vikharev. Phys. Met. Metallogr. 110, $200 \quad$ (2010). DOI: 10.1134/S0031918X10090024

14. M.P. Kashchenko, V.G. Chashchina, S. V. Vikharev. Phys. Met. Metallogr. 110, $305 \quad$ (2010). DOI: 10.1134/S0031918X10100017

15. M.P. Kashchenko, V.G. Chashchina. Dynamic Theory of $\gamma-\alpha$ Transformation in Iron Based Alloys. Solving the Problem of the Formation of Twinned Martensite Crystals. Lambert Academic., Saarbrucken, Germany (2012) 110 p.

16. M.P. Kashchenko, V.G. Chashchina. Materials Science Forum. 738-739, 3 (2013). DOI: 10.4028/www.scientific.net/MSF.738-739.3

17. M. P. Kashchenko, V. G. Chashchina. Phys. Met. Metallogr. 114, 821 (2013). DOI 10.1134/S0031918X1308005X

18. M.P. Kashchenko, V.G. Chashchina. Phys. Mesomech. 19, 107 (2016). DOI: 10.1134/S0031918X17040056

19. M.P. Kashchenko, I.F. Latypov, V.G. Chashchina. Letters on materials. 7(2), $146 \quad$ (2017). DOI: $10.22226 / 2410-3535-2017-2-146-150$

20. M. P. Kashchenko, V. G. Chashchina. Phys. Met. Metallogr.
118(4), 311 (2017). DOI: 10.1134/S0031918X17040056

21. M.P. Kashchenko, N.M. Kashchenko, V.G. Chashchina. Phys. Met. Metallogr. 118(1), $311 \quad$ (2018). DOI: 10.1134/S0031918X1801009X

22. O. M. Ivasishin, N. S. Kosenko, S. V. Shevchenko. J. Phys. IV. 5, 1017 (1995).

23. G.V. Kurdyumov, L.M. Utevskii, R.I. Entin. Transformations in iron and steel. Moscow, Nauka (1977) 238 p. (in Russian)

24. H. Warlimont, L. Delaey. Martensitic Transformations in Copper-, Silver-, and Gold-Based Alloys. Oxford, Pergamon Press (1974).

25. V.G. Pushin, V.V. Kondratyev, V.N. Khachin. Pretransition phenomena and martensitic transformations. Ekaterinburg, UrO RAN (1998) 368 p. (in Russian)

26. V.G. Pushin, E.S. Belosludtseva, V.A. Kazantsev, N. I. Kourov. Inorganic Materials: Applied Research. 4(4), 340 (2013). DOI: 10.1134/S2075113313040084

27. M. P. Kashchenko, V. G. Chashchina. Phys. Met. Metallogr. 105(6), 537 (2008). DOI: 10.1134/S0031918X08060021

28. M. P. Kashchenko, V. G. Chashchina. Phys. Met. Metallogr. 106(1), 14 (2008). DOI: 10.1134/S0031918X0807003X 